\title{
The Usefulness of Prognostic Nutritional Index in Predicting Infection in Patients with Newly Diagnosed Pancreatic Cancer
} \author{
Sarićs \\ ${ }^{1}$ Faculty of Medicine of the Military Medical Academy, University of Defence, Serbia \\ ${ }^{2}$ Clinic for Gastroenterology and Hepatology, Military Medical Academy, Serbia \\ ${ }^{3}$ Clinic for Neurosurgery, Military Medical Academy, Serbia
}

Branka Roganovićc ${ }^{1,2}$, Nebojša Manojlovićc ${ }^{1,2 *}$, Saša Perić ${ }^{1}$, Sanja Tripković ${ }^{1}$ and Ana Roganović

*Corresponding author: Nebojsa Manojlovic, Faculty of Medicine of the Military Medical Academy, University of Defence, Clinic for Gastroenterology and Hepatology, Military Medical Academy, Crnotravska 17,11000 Belgrade, Serbia

\section{ARTICLE INFO}

Received: 蔧 February 17, 2021

Published: March 01, 2021

Citation: Branka Roganović, Nebojša Manojlović, Saša Perić, Sanja Tripković, Ana Roganović Sarić. The Usefulness of Prognostic Nutritional Index in Predicting Infection in Patients with Newly Diagnosed Pancreatic Cancer. Biomed J Sci \& Tech Res 34(2)-2021. BJSTR. MS.ID.005524.

Abbreviations: PC: Pancreatic Carcinoma; CEA: Carcinoembryonic Antigen; PNI: Prognostic Nutritional Index; BMI: Body Mass Index; IQR: Interquartile Range; ROC: Receiver Operating Characteristic

\section{ABSTRACT}

Background/Aims: Data on the usefulness of prognostic nutritional index (PNI) in predicting infection in patients with pancreatic cancer before oncological treatment are limited. The aim of this study was to investigate the usefulness of PNI in predicting infection in patients with newly diagnosed pancreatic cancer.

Methods: The retrospective study included 59 adult patients. We recorded clinicopathological characteristics and laboratory analysis on admission and infectious complications that occurred during hospitalization. PNI was calculated using albumin and lymphocyte count. Association with infective complication was tested for the age, sex, body mass index, albumin levels, lymphocyte count, $\mathrm{PNI}, \mathrm{PNI}<40.5$, serum carbohydrate and carcinoembryonic antigen, tumour location, the existence of metastases and hospitalization length.

Results: In the analysed series, there were 36 (61\%) male and 23 (39\%) female patients; mean age was 62.5 years. Infectious complications were detected in $25(42,4 \%)$ patients. Three factors were found to be associated with infectious complications (Binary logistic regression): serum albumin level $(\mathrm{P}=0.019)$, PNI $(\mathrm{P}=0.030)$ and PNI below $40.5(\mathrm{P}=0.026)$. ROC curve analysis determined $\mathrm{PNI}=39.5$ as a new cut-off value for the occurrence of complications, but with relatively low sensitivity (40\%) and specificity (38.2\%).

Conclusion: Low serum albumin concentration and PNI could represent risk factors for infection in patients with newly diagnosed pancreatic cancer.

\section{Introduction}

Pancreatic Carcinoma (PC) is one of the most aggressive malignant tumours. It accounts for 458,918 new cancer cases and 432,242 cancer deaths a year worldwide [1]. Previous studies have shown that aggressive carcinomas lead to systemic inflammation and injury of the patient's immune system [2,3]. Based on this fact, several proinflammatory markers and proinflammatory scoring systems for predicting the prognosis of various malignant tumours have been defined [4-6]. Prognostic value of these scores has also been evaluated in patients with pancreatic carcinoma, especially in those with resectable tumour [7-11]. The results of several studies pointed out that the prognostic nutritional index (PNI) is an effective predictor for survival in pancreatic cancer patients after surgery and chemotherapy [12-15]. However, in the current literature there is a lack of data on the usefulness of PNI in predicting infection in patients with pancreatic cancer before starting the treatment.

This is of particular interest for the patients with newly diagnosed cancer, who might be candidates for surgical treatment or HT. Infectious complications deteriorate immune-nutritional 
status and physical function of the patients and adversely affect the onset of treatment and the course of the disease. The aim of this study was to investigate the usefulness of PNI in predicting infection in patients with newly diagnosed pancreatic cancer.

\section{Methods}

Study Design and Patient Population: The retrospective study enrolled 59 adult patients with newly diagnosed pancreatic cancer, who were treated in our clinic during a two-year period. Patients included in the study had no cancer other than pancreatic, lymphoproliferative disease and active infection on admission. They also didn't take medication that may affect lymphocyte count. The study protocol was approved by the local Ethics Committee.

Clinicopathological Characteristics: Data on clinicopathological characteristics were obtained from medical records and included age, sex, Body Mass Index (BMI), laboratory findings, tumour location (head, body, or tail of the pancreas), the existence of distant metastases (lung, liver and peritoneum) and infectious complications that occurred during hospitalization. Infectious complications included pneumonia and urinary tract infection. Blood samples for analysis were collected from each patient within $24 \mathrm{~h}$ from hospital admission and included serum albumin levels, lymphocyte counts, tumour markers: carbohydrate antigen (CA-19-9) and Carcinoembryonic Antigen (CEA). Prognostic Nutritional Index (PNI) was calculated as $10 \mathrm{x}$ albumin $(\mathrm{g} / \mathrm{dL})+$ $0.005 \times$ total lymphocyte count $\left(\right.$ per $\mathrm{mm}^{3}$ ) [16]. The lower limit for PNI was defined as 40.5. Evaluation of risk factors. The influence on the occurrence of infectious complications was tested for the age, sex, BMI, albumin levels, lymphocyte counts, PNI, PNI <40.5, CA-199 and CEA levels, tumour location, the existence of metastases and hospitalization length.

Table 1: Demographic and clinical characteristics of the patients.

\section{Statistical Analyses}

Statistical analysis was performed using the standard software package SPSS, version 19.0 (SPSS Inc., version 19.0, Chicago, IL). All continuous variables were described in the form of the median [interquartile range (IQR): range between $25^{\text {th }}$ and $75^{\text {th }}$ per centile], or mean \pm standard deviation, according to the data distribution. The normality distribution of data was tested with the KolmogorovSmirnov test. The categorical variables were expressed as absolute number (percentages) and examined using the chi-square test. Inter-group comparisons of continuous variables were performed by the non-parametric Mann-Whitney test, or the parametric Independent samples test, according to the data distribution. Binary logistic regression was used to examine demographic and clinical factors associated with the complications in the patients with pancreatic cancer. The critical value of PNI for the occurrence of infectious complications was calculated on the basis of the area under a Receiver Operating Characteristic (ROC) curve. All the analyses were evaluated at the level of statistical significance of $\mathrm{P}<0.05$.

\section{Results}

Characteristics of the Patients: We retrospectively reviewed the data of 72 patients with newly diagnosed pancreatic cancer over the two years period. Thirteen patients were excluded from the study: 6 patients did not meet inclusion criteria, 3 patients died, and 4 patients didn't have completed laboratory analysis. The data were analysed for 59 patients: 36 (61\%) male and 23 (39\%) females; mean age was 62.5 years. Infectious complications were detected in 25 (42.4\%) patients: 13 (22.1\%) patients had urinary tract infection; 12 (20.3\%) patients had pneumonia. Other characteristics of the series are presented in Table 1.

\begin{tabular}{|c|c|c|c|}
\hline Characteristics & With complications & Without complications & P value \\
\hline Number of patients & $25(42,4)$ & $34(57,6)$ \\
\hline Gender: Male / Female & $14(56,0) / 11(44,0)$ & $22(64,7) / 12(35,3)$ & $0,684^{* *}$ \\
\hline Age (years) & $62,48 \pm 12,14$ & $62,50 \pm 12,74$ & $0,995^{*}$ \\
\hline Age: $<60 / \geq 60$ & $11(44,0) / 14(56,0)$ & $14(41,2) / 20(58,8)$ & $1,000^{* *}$ \\
\hline Body Mass Index & $25,39 \pm 4,33$ & $23,97 \pm 3,53$ & $0,170^{*}$ \\
\hline Albumin g/l & $30,08 \pm 5,96$ & $33,85 \pm 5,37$ & $0,014^{*}$ \\
\hline Lymphocyte count x103/mm & $1,30(0,90-1,70)$ & $1,30(1,08-1,85)$ & $0,381^{* * *}$ \\
\hline PNI & $37,04 \pm 8,91$ & $41,77 \pm 6,77$ & $0,024^{*}$ \\
\hline PNI: $<40,5 / \geq 40,5$ & $17(68,0) / 8(32,0)$ & $13(38,2) / 21(61,8)$ & $0,046^{* *}$ \\
\hline CA19/9 & $231,30(73,15-900,50)$ & $143,55(50,38-338,75)$ & $0,111^{* * *}$ \\
\hline CEA & $3,20(1,85-11,20)$ & $3,00(2,10-7,12)$ & $0,848^{* * *}$ \\
\hline Localization: head / body / tail & $18(72,0) / 5(20,0) / 2(8,0)$ & $16(47,1) / 10(29,4) / 8(23,5)$ & $0,128^{* *}$ \\
\hline Metastasis: yes/no & $17(68,0) / 8(32,0)$ & $26(76,5) / 8(23,5)$ & $0,470^{* *}$ \\
\hline Hospitalization length (days) & $16,00(12,50-19,00)$ & $11,50(9,00-15,00)$ & $0,007^{* * *}$ \\
\hline
\end{tabular}

Note: Values are presented as an absolute number (\%), or as the mean value \pm standard deviation, or as median (interquartile range) values for the lymphocyte count, CA19/9, CEA and hospitalization lenght. *Independent Samples Test; **Chi-square test; *** MannWhitney test. 
Evaluation of Risk Factors: Clinicopathological characteristics of the patients were compared between two groups: the group with infection and the group without infection (Table 1). There was no statistically significant difference between the analysed groups for the age, sex, BMI, lymphocyte counts, CA-19-9, CEA, tumour location and existence of metastases. A significant difference was found for albumin, PNI, PNI<40.5 and length of hospitalization. The group of patients with infection had significantly lower serum albumin $(\mathrm{P}=0.014)$ and significantly longer hospitalization $(\mathrm{P}=0.007)$ than the group without infection (Table 1). PNI was significantly lower $(\mathrm{P}=0.024)$ and the prevalence of $\mathrm{PNI}<40.5$ was significantly higher (68\% vs. $38.2 \%$; P 0.046 ) in the group with infection (Table 1 \& Figures 1\&2).

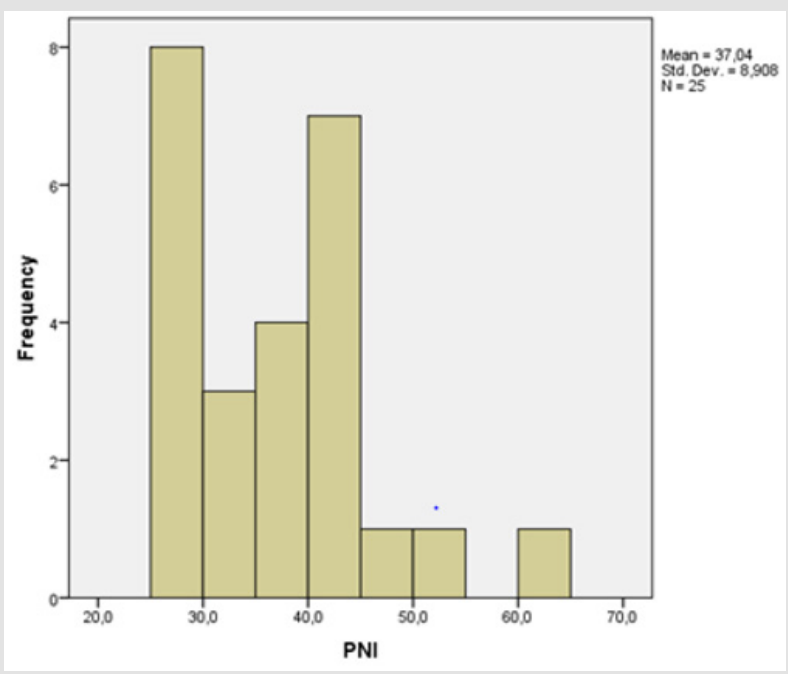

Figure 1: Distribution of PNI in a patient with complications.

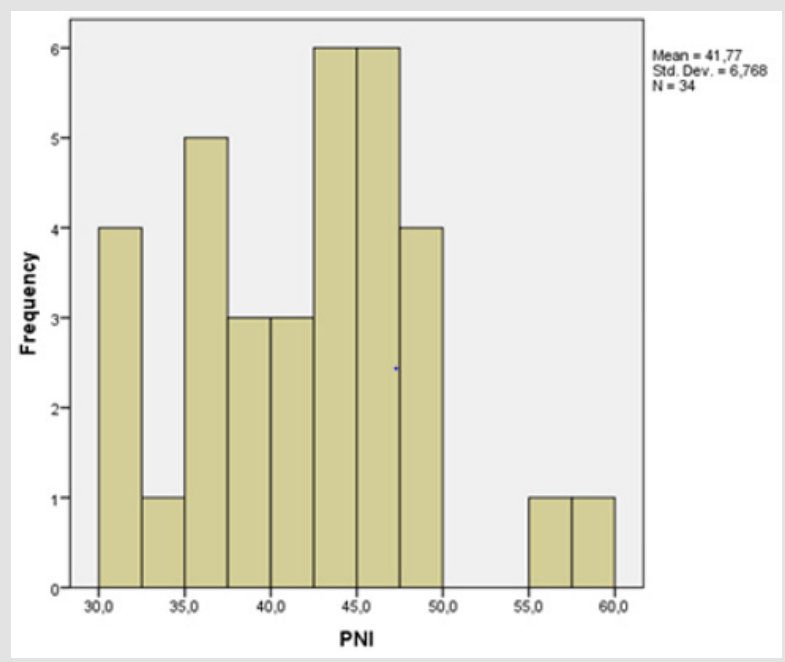

Figure 2: Distribution of PNI in a patient without complications.
Predictors of Infectious Complications: The results of binary logistic regression indicated three variables that significantly influenced the occurrence of infectious complications: serum albumin (OR:1.130, 95\%CI:1.020-1.250; P=0.019), PNI (OR:1.086, 95\% CI: 1.008-1.169; $\mathrm{P}=0.030$ ) and PNI below 40.5 (OR:3.136, 95\%CI:1.156-10.204; $\mathrm{P}=0.026)$. Patients who have lower albumin, lower PNI, and a PNI index value below 40.5 are more likely to develop infection (Table 2). The most important predictor is PNI below 40.5: patients with a PNI below 40.5 are 3.5 times more likely to get an infection, than the patients with a PNI 40.5 and greater. When ROC analysis was performed to calculate the sensitivity and specificity of the PNI, it was shown that PNI is a good indirect indicator of the occurrence of infectious complications in patients with pancreatic cancer. ROC curve analysis also determined $\mathrm{PNI}=39.5$ as a new cut-off value for the occurrence of complications, but with relatively low sensitivity (40\%) and specificity (38.2\%) (Figure 3).

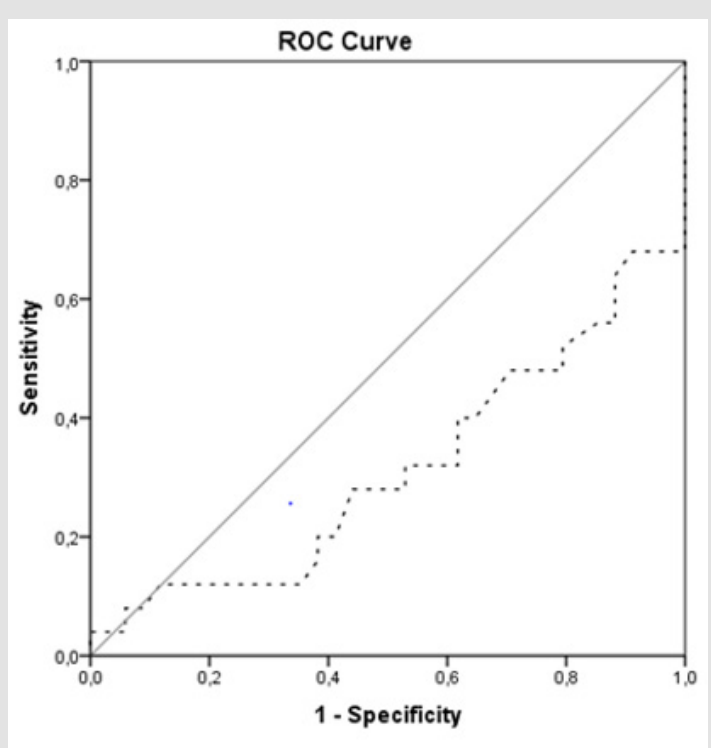

Diagonal segments are produced by ties.

Figure 3: Receiver operating characteristic curves for predicting complications with prognostic nutritional index $<39.5$.

Table 2: Predictors of infectious complications in patients with diagnosed pancreatic cancer.

\begin{tabular}{|c|c|c|}
\hline \multicolumn{3}{|c|}{ Binary logistic regression } \\
\hline Characteristics & OR (95\%CI) & P value \\
\hline Gender: Male / Female & $1,440(0,500-4,147)$ & 0,499 \\
\hline Age (years) & $1,000(0,959-1,043)$ & 0,995 \\
\hline Age: $<60 / \geq 60$ & $0,891(0,314-2,531)$ & 0,828 \\
\hline Body Mass Index & $1,103(0,958-1,270)$ & 0,173 \\
\hline Albumin $\mathrm{g} / \mathrm{l}$ & $1,130(1,020-1,250)$ & 0,019 \\
\hline Lymphocyte count $\times 10^{3} / \mathrm{mm}$ & $0,837(0,454-1,544)$ & 0,570 \\
\hline
\end{tabular}




\begin{tabular}{|c|c|c|}
\hline PNI & $1,086(1,008-1,169)$ & 0,030 \\
\hline PNI: $<40.5 / \geq 40.5$ & $3,436(1,156-10,204)$ & 0,026 \\
\hline CA19/9 & $1,000(0,999-1,001)$ & 0,853 \\
\hline CEA & $0,998(0,978-1,019)$ & 0,862 \\
\hline Localization: head / body / tail & $0,444(0,125-1,578)$ & 0,210 \\
\hline head & $2,893(0,960-8,713)$ & 0,059 \\
\hline body & $0,600(0,176-2,046)$ & 0,414 \\
\hline tail & $0,283(0,054-1,468)$ & 0,133 \\
\hline Metastasis: yes/no & $1,529(0,482-4,855)$ & 0,471 \\
\hline Hospitalization length (days) & $1,088(0,995-1,190)$ & 0,063 \\
\hline
\end{tabular}

Note: OR -Odss ratio, CI-Confidence interval. Significant values $(\mathrm{P}<0.05)$ are presented in bold.

\section{Discussion}

In the patients with malignant tumours, systemic inflammatory response plays a crucial role in the development and progression of tumours [7-20]. On the other hand, nutritional status has a significant role in the prognosis of these patients [21-23]. In 1984. Onodera and colleagues defined PNI as a simple index, based on the values of serum albumin and lymphocytes, which reflects both: the patient's nutritional and inflammatory status [16]. PNI was originally established to estimate the operative risk in patients with malignant gastrointestinal tumours. Onodera's results indicated that gastrointestinal surgery can be safely performed when the PNI is over 45.16 Studies carried out on the following years reported that low preoperative PNI is a poor prognostic factor for overall survival in patients with different types of human malignant tumours [2434]. Considering pancreatic cancer, many authors have identified low PNI as a prognostic factor for shorter survival in patients with resectable disease and in those who underwent chemotherapy, or chemoradiotherapy for unresectable cancer [15,35-39].

Furthermore, Kim NH demonstrated that PNI below 45 is significantly associated with early recurrence after curative surgical resection [40]. We investigated the significance of PNI in predicting infection in patients with pancreatic carcinoma before starting the therapy - chemotherapy or/and surgery. According to our results, low PNI, especially PNI below 40.5 on hospital admission was significantly associated with the occurrence of infectious complications during hospitalization. The calculated critical value of PNI for the occurrence of infectious complications was 39.5. These results are partly consistent with the results of other authors, although an adequate comparison is not possible, due to the different design of the studies. Namely, studies on the prognostic significance of PNI for the occurrence of complications in patients with various carcinomas were mainly conducted in the patients who underwent surgery. In general, most authors agree that low preoperative PNI can predict postoperative complications, such as pneumonia, urinary and wound infection, anastomotic leakage, bleeding [41-47].
Some studies have shown that pancreatic cancer patients with a low preoperative PNI, or with PNI reduction postoperatively, have a higher risk of complications after pancreaticoduodenectomy $[48,49]$. Narongsak et college demonstrated that early postoperative PNI below 40.5 is a significant predictive factor for postoperative infective complications such as intra-abdominal abscess, surgical site infection, pneumonia, septicaemia and urinary tract infection [50]. In the study of Watanabe PNI below 40 was significantly associated with postoperative pneumonia, while Kanda et al pointed to PNI cut-off value 45 as the prognostic factor for the complications after pancreaticoduodenectomy11,48 Our PNI cut-off value of 39.5 is lower than the cut off values in previous studies, possibly due to the fact that this study included patients before surgery or chemotherapy. It should also be mentioned that some studies have not confirmed the predictive usefulness of PNI for the occurrence of postoperative complications, especially intraabdominal infections and pancreatic fistula [51-53].

The results of the present study indicate that, beside PNI, low serum albumin is a predictor of infectious complications in patients with newly diagnosed pancreatic carcinoma. This result is not surprising, since albumin does not only reflect nutritional, but also inflammatory and immunological status [54,55]. In malignant diseases, hypoalbuminemia is a consequence of the increased demand for amino acids, and increased microvascular permeability, due to the action of inflammatory cytokines and, on the other hand, shortened albumin half-life [54-56]. Several studies have indicated that in patients with gastrointestinal malignancies, hypoalbuminemia is a good predictor for postoperative complications [48,50,57-59]. Narongsak et al. reported that low preoperative serum albumin is a significant predictive factor for serious infective complications after pancreaticoduodenoctomy [50].

In the Augustine study, preoperative hypoalbuminemia was pointed as an independent risk factor for Clavien-Dindo grade IV complication and mortality after pancreatic surgery [60]. Furthermore, there is evidence that postoperative decrease of serum albumin is a risk factor for the occurrence of complications after abdominal surgery $[60,61]$. According to the cited studies, it is clear that hypoalbuminemia is a good predictor for infection in patients with pancreatic carcinoma, especially after pancreatic resection. Our result pointed hypoalbuminemia as a risk factor for infection in these patients even before surgery, or chemotherapy.

\section{Limitation of the Study}

This study has some limitations. First: Due to the retrospective design of the study, there may have been a certain degree of selection bias. Second: A small number of patients were included in the study. Third: With respect of relatively low sensitivity and specificity for PNI cut off value of 39.5, this result should be accepted with 
caution. Further investigations are needed to test its significance in clinical practice.

\section{Conclusion}

This study showed that serum albumin concentration and PNI are significant factors associated with infection in patients with pancreatic carcinoma. Infectious complications can postpone the onset of treatment and adversely affect the course of the disease. Therefore, patients with low serum albumin and PNI on hospital admission should be closely followed up during hospitalization and early intervention with immuno-nutrition should be considered.

\section{References}

1. Bray F, Ferlay J, Soerjomataram I, Siegel RL, Torre LA, et al. (2018) Global cancer statistics 2018: GLOBOCAN estimates of incidence and mortality worldwide for 36 cancers in 185 countries. CA Cancer J Clin 68(6): 394 424.

2. Sideras K, Braat H, Kwekkeboom J, Van Eijck CH, Peppelenbosch MP, et al. (2014) Role of the immune system in pancreatic cancer progression and immune modulating treatment strategies. Cancer Treat Rev 40: 513522.

3. Bazhin AV, Shevchenko I, Umansky V, Werner J, Karakhanova S (2014) Two immune faces of pancreatic adenocarcinoma: possible implication for immunotherapy. Cancer Immunol Immunother 63(1): 59-65.

4. Lee JY, Kim HI, Kim YN, Hong JH, Alshomimi S, et al. (2016) Clinical Significance of the Prognostic Nutritional Index for Predicting Shortand Long-Term Surgical Outcomes After Gastrectomy: A Retrospective Analysis of 7781 Gastric Cancer Patients. Medicine (Baltimore) 95(18): e3539.

5. Peng J, Zhanq R, Zhao Y, Wu X, Chen G, et al. (2017) Prognostic value of preoperative prognostic nutritional index and its associations with systemic inflammatory response markers in patients with stage III colon cancer. Chin J Cancer 36(1): 96.

6. Mei Z, Shi L, Wang B, Yang J, Xiao Z, et al. (2017) Prognostic role of pretreatment blood neutrophil-to lymphocyte ratio in advanced cancer survivors: A systematic review and meta-analysis of 66 cohort studies. Cancer Treat Rev 58: 1-13.

7. Asaoka T, Miyamoto A, Maeda S, Tsujie M, Hama N, et al. (2016) Prognostic impact of preoperative NLR and CA19-9 in pancreatic cancer Pancreatology 16(3): 434-440.

8. Tsujita E, Ikeda Y, Kinjo N, Yamashita YI, Hisano T, et al. (2017) Postoperative Neutrophil-to-Lymphocyte Ratio as a Predictor of LongTerm Prognosis after Pancreatectomy for Pancreatic Carcinoma: A Retrospective Analysis. Am Surg 83(6): 610-166.

9. Hoshimoto S, Hishinuma S, Shirakawa H, Tomikawa M, Ozawa (2019) Validation and clinical usefulness of pre- and postoperative systemic inflammatory parameters as prognostic markers in patients with potentially resectable pancreatic cancer. Pancreatology S14243903(19): 30801-30804.

10. Suqimachi K, Iquishi T, Mano Y, Nishijima T, Nakanoko T, et al. (2019) The Impact of Immunonutritional and Physical Status on Surgical Outcome After Pancreaticoduo-denectomy in Elderly Patients. Anticancer Res 39(11): 6347-6353.

11. Watanabe J, Otani S, Sakamoto T, Arai Y, Hanaki T, et al. (2016) Prognostic indicators based on inflammatory and nutritional factors after pancreaticoduodenectomy for pancreatic cancer. Surg Today 46(11): 1258-1267.

12. Nakagawa K, Sho M, Akahori T, Nagai M, Nakamura K, et al. (2019) Significance of the inflammation-based prognostic score in recurrent pancreatic cancer. Pancreatology 19(5): 722-728.
13. Li S, Tian G, Chen Z, Zhuanq Y, Li G (2019) Prognostic Role of the Prognostic Nutritiona Index in Pancreatic Cancer: A Meta-analysis. Nutr Cancer 71(2): 207-213.

14. Abe T, Nakata K, Kibe S, Mori Y, Miyasaka Y, et al. (2018) Prognostic Value of Preoperative Nutritional and Immunological Factors in Patients with Pancreatic Ductal Adenocarcinoma. Ann Surg Oncol 25(13): 3996-4003.

15. Liu J, Jianq S, Yanq X, Li X, Wanq N (2018) The Significant Value of Preoperative Prognostic Nutritional Index for Survival in PancreaticCancers: A Meta-analysis. Pancreas 47(7): 793-799.

16. Onodera T, Goseki N, Kosaki G (1984) Prognostic nutritional index in gastrointestinal surgery of malnourished cancer patients. Nihon Geka Gakkai Zasshi 85(9): 1001-1005.

17. Roxburgh CS, McMillan DC (2010) Role of systemic inflammatory response in predicting survival in patients with primary operable cancer. Future Oncol 6(1): 149-163.

18. Giraldo NA, Becht E, Vano Y, Sautes Fridman C, Fridman WH (2015) The immune response in cancer: from immunology to pathology to immunotherapy. Virchows Arch 467(2): 127-135.

19. Lin J, Fang T, Zhu M, Xu X, Zhang J, et al. (2019) Comparative performance of inflammation-based prognostic scores in patients operated for intrahepatic cholangiocarcinoma. Cancer Managment and Research 11: 9107-9019.

20. Dogan M, Algin E, Guven ZT, Baykara M, Kos TF, et al. (2018) Neutrophillymphocyte ratio, platelet-lymphocyte ratio, neutrophil-platelet score and prognostic nutritional index: do they have prognostic significance in metastatic pancreas cancer? Curr Med res Opin 34(5): 857-863.

21. Ramos R, Nadal E, Peiro I, Masuet Aumatell C, Macia I, et al. (2018) Preoperative nutritional status assessment predicts postoperative outcomes in patients with surgically resected non-small cell lung cancer. Eur J Surg Oncol 44(9): 1419-1424.

22. Schutte K, Tippelt B, Schulz C, Rohl FW, Feneberg A, et al. (2015) Malnutrition is a prognostic factor in patients with hepatocellular carcinoma (HCC). Clin Nutr 34(6): 1122-1127.

23. Gheorghe C, Pascu O, Iacob R, Vadan R, Iacob S, et al. (2013) Nutritional risk screening and prevalence of malnutrition on admission to gastroenterology departments: a multicentric study. Chirurqia (Bucur) 108(4): 535-541.

24. Li P, Wang X, Lai Y, Zhou K, Tang Y (2019) The prognostic value of pretreatment prognostic nutritional index in esophageal squamous cell carcinoma: A meta-analysis. Medicine (Baltimore) 98(22): e15280.

25. Zhang L, Su Y, Chen Z, Wei Z, Han W (2017) The prognostic value of preoperative inflammation-based prognostic scores and nutritional status for overall survival in resected patients with nonmetastatic Siewert type II/III adenocarcinoma of esophagogastric junction. Medicine (Baltimore) 96: e7647.

26. Nakatani M, Migita K, Matsumoto S, Wakatsuki K, Ito M, et al. (2017) Prognostic significance of the prognostic nutritional index in esophageal cancer patients undergoing neoadjuvant chemotherapy. Dis. Esophagus 30(8): 1-7.

27. Kosuga T, Konishi T, Kubota T, Shoda K, Konishi H, et al. (2019) Value of Prognostic Nutritional Index as a Predictor of Lymph Node Metastasis in Gastric Cancer.Anticancer Res 39(12): 6843-6849.

28. Gao J, Wang Y, Li F, Zhu Z, Han B, et al. (2019) Prognostic Nutritional Index and Neutrophil-to-Lymphocyte Ratio Are Respectively Associated with Prognosis of Gastric Cancer with Liver Metatasis Undergoing and without Hepatectomy. Biomed Res int.

29. Migita K, Takayama T, Saeki K, Matsumoto S, Wakatsuki K, et al. (2013) The prognostic nutritional index predicts long-term outcomes of gastric cancer patients independent of tumor stage. Ann Surg Oncol 20(8): 2647-2654. 
30. Pinato DJ, North BV, Sharma R (2012) A novel, externally validated inflammation-based prognostic algorithm in hepatocellular carcinoma the prognostic nutritional index (PNI). Br J Cancer 106(8): 1439-1445.

31. Jian Hui C, Iskandar EA, Cai Shi, Chen CQ (2016) Significance of Onodera's prognostic nutritional index in patients with colorectal cancer: a large cohort study in a single Chinese institution. Tumour Biol 37(3): 32773283.

32. Sun G, Li Y, Peng Y, Lu D, Zhang F, et al. (2019) Impact of the preoperative prognostic nutritional index on postoperative and survival outcomes in colorectal cancer patients who underwent primary tumor resection: a systematic review and meta-analysis. Int J Colorectal Dis 34(4): 681-689.

33. Peng J, Zhang R, Zhao Y, Wu X, Chen G, et al. (2017) Prognostic value of preoperative prognostic nutritional index and its associations with systemic inflammatory response markers in patients with stage III colon cancer. Chin J Cancer 36(1): 96.

34. Hong S, Zhou T, Fang W, Xue C, Hu Z, et al. (2015) The prognostic nutritional index (PNI) predicts overall survival of small-cell lung cancer patients.Tumour Biol 36(5): 3389-3397.

35. Lee SH, Chunq MJ, Kim B, Lee HJ, Heo JY, et al. (2017) The Significance of the Prognostic Nutritional Index for All Stages of Pancreatic Cancer. Nutr Cancer 69(3): 512-519.

36. Nakagawa S, Yamashita YI, Umezaki N, Yamao T, Okabe H, et al. (2018) Serum Marker Score Based on Prognostic Nutrition Index Carcinoembryonic Antigen, and Carbohydrate Antigen 19-9 Is Associated with Recurrence for Patients Undergoing Surgery for Pancreatic Ductal Adenocarcinoma. Pancreas 47(9): 1130-1134.

37. Geng Y, Qi Q Sun M, Chen H, Wang P (2015) Prognostic nutritional index predicts survival and correlates with systemic inflammatory response in advanced pancreatic cancer. Eur J Surg Oncol 41(11): 1508-1514.

38. Shimizu T, Taniguchi K, Asakuma M, Tomioka A, Inoue Y, et al. (2019) Lymphocyte-to-Monocyte Ratio and Prognostic Nutritional Index Predict Poor Prognosis in Patients on Chemotherapy for Unresectable Pancreatic Cancer. Anticancer Res 39(4): 2169-2176.

39. Abe T, Nakata K, Kibe S, Mori Y, Miyasaka Y, et al. (2018) Prognostic Value of Preoperative Nutritional and Immunological Factors in Patients with Pancreatic Ductal Adenocarcinoma. Ann Surg Oncol 25(13): 3996-4003.

40. Kim KH, Hwanq HK, Kanq IC, Lee WJ, Kang CM (2019) Oncologic impact of preoperative prognostic nutritional index change in resected pancreatic cancer following neoadjuvant chemotherapy. Pancreatology 20(2): 247-253.

41. Satoru O, Junichi S, Satoshi T, Daishiro K, Hiraoaki T, et al. (2018) Risk Stratification According to the Prognostic Nutritional Index for Predicting Postoperative Complications After Lung Cancer Surgery. Ann Surg Oncol 25(5): 1254-1261

42. Yang Y, Gao P, Song Y, Sun J, Chen X, et al. (2016) The Prognostic Nutritional Index Is a Predictive Indicator of Prognosis and Postoperative Complications in Gastric Cancer: A Meta-Analysis. Eur ] surg Oncol 42(8): 1176-1182.

43. Katsunobu S, Masaichi O, Tatsuro T, Takahiro T, Ryosuke A, et al. (2016) Predictive Potential of Preoperative Nutritional Status in Long-Term Outcome Projections for Patients with Gastric Cancer. Ann Surg Oncol 23(2): 525-533.

44. Masano S, Hajime Y, Kazuhiko Y, Yuki Y, Sachiyo O, et al. (2018) The Influence of Immunity, Nutrition, and Physical Function on the Onset of Pneumonia After Colorectal Cancer Resection. Gan To Kagaku Ryoho 45(10): 1486-1488.

45. Mengyun Ke, Tao Xu, Na Li, Yifan Ren, Aihua Shi, et al. (2016) Prognostic Nutritional Index Predicts Short-Term Outcomes After Liver Resection for Hepatocellular Carcinoma Within the Milan Criteria. Oncotarget 7(49): 81611-8120.
46. Bailon Cuadrado M, Perez Saborido B, Sanchez Gonzalez J, Rodriguez Lopez M, Velasco Lopez R, et al. (2019) Prognostic Nutritional Index predicts morbidity after curative surgery for colorectal cancer. Cir Esp 97(2): 71-80.

47. Borda F, Miranda C, Borda A, Echeverría E, Guerra A, et al. (2017) [Relation between preoperative prognostic Onodera's Index and postsurgery complications in the R0 gastric carcinoma resection]. An Sist Sanit Navar 40(1): 67-75

48. Kanda M, Fujii T, Kodera Y, Nagai S, Takeda S (2011) Nutritional predictors of postoperative outcome0 in pancreatic cancer. Br J Surg 98(2): 268-274

49. Norihiro Sato, Yasuhisa Mori, Noritaka Minagawa, Toshihisa Tamura, Kazunori Shibao (2014) Rapid Postoperative Reduction in Prognostic Nutrition Index Is Associated with the Development of Pancreatic Fistula Following Distal Pancreatectomy. Pancreatology 14(3): 216-220.

50. Narongsak R, Pongsatorn T, Wikran S, Paramin M, Somkit M (2019) Correlation of serum albumin and prognostic nutritional index with outcomes following pancreaticoduodenectomy. World J Clin Cases 7(1): 28-38.

51. Atsushi N, Masahide H, Naoya I, Koichi Y, Takeomi H, et al. (2019) Clinical Significance of Preoperative Nutritional Parameter and Patient Outcomes After Pancreatectomy: A Retrospective Study at Two Academic Institute. Ann Hepatobiliary Surg 23(2): 168-173.

52. Wang DS, Luo HY, Qiu MZ, Wang ZQ, Zhang DS, et al. (2012) Comparison of the prognostic values of various inflammation-based factors in patients with pancreatic cancer. Med Oncol 29(5): 3092-3100.

53. Ikeguchi M, Hanaki T, Endo K, Suzuki K, Nakamura S, et al. (2017) C-Reactive Protein/Albumin Ratio and Prognostic Nutritional Index Are Strong Prognostic Indicators of Survival in Resected Pancreatic Ductal Adenocarcinoma. J Pancreat Cancer 3(1): 31-36.

54. Soeters BP, Wolfe RR, Shenkin A (2019) Hypoalbuminemia: Pathogenesis and Clinical Significance. JPEN J Parenter Enteral Nutr 43(2): 181-93.

55. Rai T, Wu X, Shen B (2015) Frequency and risk factors of low immunoglobulin levels in patients with inflammatory bowel disease. Gastroenterol Rep (Oxf) 3(2): 115-121.

56. OGorman P, McMillan D, McArdle CS (1998) Impact of weight loss, appetite, and the inflammatory response on quality of life in gastrointestinal cancer patients. Nutr Cancer 32: 76-80.

57. Costa MD, Vieira de Melo CY, Amorim AC, Cipriano Torres Dde O, Dos Santos AC (2016) Association Between Nutritional Status, Inflammatory Condition, and Prognostic Indexes with Postoperative Complications and Clinical Outcome of Patients with Gastrointestinal Neoplasia. Nutr Cancer 68(7): 1108-1114.

58. Bozzetti F, Gianotti L, Braga M, Carlo DV, Mariani L (2007) Postoperative Complications in Gastrointestinal Cancer Patients: The Joint Role of the Nutritional Status and the Nutritional Support. Clin NUtr 26(6): 698709.

59. Hennessey BD, Burke PJ, Ni Dhonochu T, Shields C, Winter CD, et al. (2010) Preoperative hypoalbuminemia is an independent risk factor for the development of surgical site infection following gastrointestinal surgery: a multi-institutional study. Ann Surg 252(2): 325-329.

60. Augustin T, Burstein MD, Schneider EB, Morris Stiff G, Wey J, et al. (2016) Frailty predicts risk of life-threatening complications and mortality after pancreatic resections. Surgery 160(4): 987-996.

61. Labgaa I, Joliat GR, Kefleyesus A, Mantziari S, Schäfer M, et al. (2017) Is postoperative decrease of serum albumin an early predictor of complications after major abdominal surgery? A prospective cohort study in a European centre. BMJ Open 7(4): e013966. 
ISSN: 2574-1241

DOI: 10.26717/BJSTR.2021.34.005524

Nebojša Manojlović. Biomed J Sci \& Tech Res

(c) (P) This work is licensed under Creative

Submission Link: https://biomedres.us/submit-manuscript.php

$\begin{array}{ll}\text { BIOMEDICAL } & \text { Assets of Publishing with us } \\ \text { RESEARCHES } & \text { Global archiving of articles } \\ \text { - Immediate, unrestricted online access }\end{array}$

